\title{
Guest editors' preface to special issue on interval temporal logics
}

\author{
Ben Moszkowski · Dimitar Guelev • Martin Leucker
}

Published online: 10 April 2014

(C) Springer International Publishing Switzerland 2014

This special issue contains a selection of revised and expanded versions of papers originally presented in a special track on Interval Temporal Logics (ITLs) at the 18th International Symposium on Temporal Representation and Reasoning (TIME 2011). The conference was held in Lübeck, Germany on 12-14 September, 2011, and another special track on ITLs is planned for TIME 2014 in Verona, Italy. The articles reflect the varied nature of research on the theory and application of ITLs. Two aims of this preface are to discuss the study of ITLs, and in the context of that to give summaries of the articles.

A previous journal special issue on ITLs appeared about ten years ago. In its preface [12] the guest editors Valentin Goranko and Angelo Montanari pointed out that the concept of time intervals is naturally appealing and intuitive, with research on associated logical formalisms having compelling multidisciplinary origins arising from Philosophy, Linguistics, Artificial Intelligence and Computer Science. This has certainly helped in no small measure to perpetuate the study of ITLs and ensure that the investigation of their theory and application continues to be seen as worthwhile. As a reflection of the numerous sources of motivation, the earlier special issue contained an extensive "roadmap" survey of such formalisms by Goranko, Montanari and Sciavicco [13]. Nevertheless, research on ITLs has continued to face major hurdles already noted in [12]. These result from two kinds of complexity often exhibited by the notations:

B. Moszkowski ( $\square)$

Software Technology Research Laboratory, De Montfort University, Leicester, UK

e-mail: benm@dmu.ac.uk

D. Guelev

Department of Algebra and Logic, Institute of Mathematics and Informatics, Sofia, Bulgaria

e-mail: gelevdp@math.bas.bg

M. Leucker

Institute for Software Engineering and Programming Languages, University of Lübeck, Lübeck, Germany

e-mail: leucker@isp.uni-luebeck.de 
- Conceptual and technical complexity: ITLs can seem relatively hard to learn, understand and use in comparison to conventional point-based Propositional Linear-Time Temporal Logic (PLTL) [24, 29] and other frameworks.

- Computational complexity: Both validity and model checking, if decidable at all, are often of nonelementary complexity for propositional ITLs.

A reluctance to use ITLs because of these concerns can involve some arguably questionable subjectivity. For example, formulas in well-known point-based temporal logics with the same expressiveness can in fact be quite low-level and more obscure than equivalent interval-based ones. The second concern generally has to do with worst-case computational complexity, which is judiciously avoidable in various successful applications. Interestingly, Quantified Propositional Linear-Time Temporal Logic (QPLTL) [27] and linear-time $\mu$ calculus [2], which both share propositional ITL's expressive power, also have problems involving complexity. Hence, evaluating how two kinds of logics fare on the same relevant classes of properties is much more commonsense. Then the greater expressive power of ITLs when compared, for instance, with discrete-time PLTL becomes apparent. ${ }^{1}$ Consider Dam's remarks in [6] about a telling exercise featuring some plain looking illustrative $\mu$ calculus formulas: Even though it may seem very natural to describe temporal properties by extremal fixed points, the linear time $\mu$-calculus ([like] other $\mu$-calculi) is nonetheless difficult to use in practice. This is true, in particular, when fixed points alternate.

The natural and convenient interval-based sequential composition of formulas using the chop connective stands out for various kinds of applications (e.g., see Schellhorn et al.'s article in the special issue). When proposing a logic with a somewhat different form of chop, Müller-Olm [33] states: Indeed, at first glance there seems to be no natural way for explaining the meaning of sequentially composed formulae $\phi_{1} ; \phi_{2}$ [using chop] in the setting of point-based temporal or modal logic, as there is no natural notion for where [the] interpretation of $\phi_{1}$ stops and [the] interpretation of $\phi_{2}$ starts.

Below is a brief summary of some trends and promising developments over the last ten years:

- An extensive research programme systematically classifies an important group of ITLs [7].

- An IEEE standard [18] is approved which contains an executable assertion language influenced by ITLs.

- A research monograph [37] and a textbook [34] make extensive use of ITLs, and other modern textbooks $[10,24]$ include material on ITLs in increasing detail.

- Multi-core processors become ubiquitous as technological limits for clock rates are being reached. Research on ITLs (e.g., [4]) suggests they might offer a promising general framework for formal reasoning about some relevant concurrent algorithms involving fine-grained interleaved access to shared variables.

- A revised standard SQL:2011 for the database Structured Query Language (SQL) now includes support for time intervals $[19,25]$.

In what follows, some connections between the trends, developments and topics covered by the special issue's articles will be noted.

\footnotetext{
${ }^{1}$ Lichtenstein et al. [27], Moszkowski [30] (reproduced in [32]) and Kröger and Merz [24, Chaps. 3-4] provide comparisons of various logics' expressiveness.
} 
One consequence of the complexity issues associated with ITLs is that research on model-checking techniques for these formalisms has been comparatively limited. This is in stark contrast to the significant and widespread take-up by academia and industry of model checking with properties expressed in point-based temporal logics. Nevertheless, ITLs have influenced the notation for executable assertions in IEEE Standard 1647 [18] for the language $e$ (approved by the IEEE in 2006, with revisions in 2008 and 2011). ${ }^{2}$

Executable subsets of ITLs have been successfully employed in a number of applications besides the IEEE standard just mentioned. For example, according to Schellhorn et al.'s article in the special issue involving symbolic execution, lower-level point-based temporal logics (such as those advocated by Manna and Pnueli [29] and Lamport [26]), while certainly excellent for model checking finite-state systems, seem less appropriate for deductive reasoning about many practical shared-variable concurrent algorithms with relevance to multi-core processors. Given the present widespread availability of such hardware in even relatively inexpensive handheld devices, the desirability of suitable general formal frameworks for the associated subtle concurrent algorithms might become a significant practical motivation for ITLs. Whatever the future holds, it is certainly remarkable that over the last ten years ITLs have become serious contenders for verification of shared-variable concurrency, with at least four research groups currently pursuing this topic.

Difficulties similar to those experienced when using point-based temporal logics to reason about various shared-variable algorithms with fine-grained concurrency also appear relevant to Process Algebras, which have certainly been quite successful for various other applications. Following Hoare's presentation over five years ago of a promising research plan [17] particularly motivated by the emergence of multi-core processors, progress with using Process Algebras to help verify such algorithms has been limited. Of course, the situation could ultimately change.

On the other hand, a recent example of persistent concerns by many researchers of ITLs' conceptual complexity involves RGITL [4], which combines Jones' rely-guarantee conditions [22] with Moszkowski's ITL [31]. RGITL is the subject of Schellhorn et al.'s article in this special issue and is used to mechanically verify algorithms with shared-variable concurrency. Jones observes in [23] that RGITL could perhaps be quite attractive ("seductive" in his words) for that, although it might be too expressive, particularly when used by an unskilled person. Therefore, more evaluation and comparison will be needed to determine whether such a powerful and general interval-based framework is overkill in relation to approaches specifically developed for the required purposes.

In this short survey it seems reasonable to divide temporal formalisms for intervals into four main categories:

1. Non-modal calculi for Allen's seminal binary relations between intervals [1].

2. Other algebraic notations and also those based on first- or higher-order logics with variables explicitly denoting intervals or sets of intervals.

3. Modal formalisms such as Halpern-Shoham logic (HS-logic) [15] and Venema's $C D T$ [36] which have interval-based variables and can express Allen's relations.

4. Moszkowski's Interval Temporal Logic (ITL) [20, 31], and variants and adaptations of it which make significant use of state-based variables as well as the temporal operator

\footnotetext{
${ }^{2}$ The standard's introduction includes the following statement: " $e$ contains a temporal language that borrows from Linear Temporal Logic and Interval Temporal Logic."
} 
chop which sequentially composes two arbitrary formulas. Such formalisms include the Duration Calculus (DC) [37, 38] for reasoning about real-time and hybrid systems.

Notations in the first two non-modal categories typically have variables denoting either intervals (e.g., represented as primitive objects or as pairs of endpoints) or sets of intervals. Such formalisms have been used in Artificial Intelligence and Databases (e.g., SQL:2011's temporal intervals mentioned above). The article in the special issue by Combi and Sala applies Allen's relations with variables denoting sets of intervals to Databases. Golumbic and Shamir's influential graph-theoretic analysis of Allen relations and algorithms for them [11] has helped to popularise the framework.

The modal perspective offered by the third and fourth categories makes intervals implicit. The logics express properties about the intervals but without variables to denote them. All of the special issue's articles besides Combi and Sala's deal with such modal formalisms. The notations in the two modal categories have some overlap since, for example, both CDT and ITL have the chop operator. However, significant and long-standing diversity of research interests and focus is found in work involving these categories. It is reflected in the special issue and now discussed.

Researchers of the third category's HS-logic and other such modal frameworks which can express Allen's relations generally eschew connections with point-based temporal logics, instead preferring so-called pure, interval-based variables. The pure notations are also known as nonlocal and in practice are almost always propositional. In contrast, variables in the fourth category's notations are usually local, that is, they concern behaviour over states. Only the local formalisms' formulas and expressions involve interval behaviour. Furthermore, unlike research in the third category, which primarily concentrates on studying the quite challenging theory of pure propositional logics, work in the fourth category considers propositional, first-order and higher-order ITLs with state-based variables. However, there has been some limited use of pure, interval-based variables in connection with DC, as noted below.

Della Monica et. al in [7] survey an extensive and productive research programme carried out for over ten years to classify the expressiveness of pure sublogics of HS-logic for various models of time, and also to determine computational complexity, which in many cases amounts to proving undecidability. ${ }^{3}$ The associated website [21] includes an online tool for examining results of this research, and a general bibliography about ITLs. In contrast to non-modal characterisations of Allen's relations, pure modal varieties have so far had little practical take-up in applications in the targeted areas of Artificial Intelligence and Databases. Bresolin et al. [5] seek to address this by presenting some early experience with a newly implemented decision procedure for one HS-sublogic (accessible online via [21]). Lomuscio and Michaliszyn [28] have been recently investigating extensions of HS-logic and associated algorithms intended for model checking multi-agent systems.

Unlike the third category involving pure ITLs, formulas in the fourth category, especially for practical applications, generally involve local, state-based variables. The fourth category is therefore much closer to conventional point-based temporal logics than the third one is. Indeed, point-based operators and properties can be readily expressed, as is commonly done. Propositional systems involving only local variables are generally decidable, but can involve nonelementary complexity (e.g., an early result by Kozen is mentioned in [30] and

\footnotetext{
${ }^{3}$ In spite of survey's general title “Interval temporal logics: a journey”, only progress in research involving pure formalisms is considered.
} 
reproduced in [32]). Work on such formalisms has provided a steady stream of applications and software tools (e.g., see the special issue's article by Schellhorn et al.). Publications include Zhou and Hansen's extensive research monograph [37] and Olderog and Dierks' textbook on real-time systems [34]. These two books employ DC as the main logical notation, and the latter contains many exercises. Zhou and Hansen include a discussion of some connections between Allen's relations, HS-logic and an alternatively derived version of DC described earlier by Roy and Zhou [35] with state-based variables as in conventional DC.

Recent textbooks on temporal logics by Kröger and Merz [24] and Fisher[10] have material about local ITLs. Fisher also examines Allen's influential interval relations and the literature on them. Local formalisms have influenced the IEEE standard already mentioned, the HOL theorem prover [14] and executable subsets of point-based temporal logics such as Metatem [3] (see also [10, Chap. 6]).

As has already been emphasised above, ITL and DC, unlike HS-logic, are routinely used with local, state-based variables. However, there is an interesting diversity of opinion about the significance of nonlocal variables in DC. The issue centres on durations, which are interval-oriented DC expressions of the form $\int e$, for some state-based expression $e$. The value of a duration $\int e$ for a temporal interval equals the integral of $e$ 's value over that interval (e.g., $\int 1$ equals the interval's length). Durations are considered by Hansen and Zhou [16, 37] to be pure, nonlocal variables, and are referred to as temporal variables with structure. There are at present differing views about whether DC's durations should indeed be formally classified as nonlocal variables, but the details are outside the scope of this survey. However, conventional nonlocal variables occur in a variant of first-order ITL previously introduced by Dutertre [9] and motivated by DC. Hansen and Zhou [16, 37] prove relative completeness of an axiom system for DC by employing a reduction to firstorder ITL on the reals. Connections between the nonlocal ITL variables and DC's durations are instrumental in Hansen and Zhou's proof.

The dichotomy between pure and local ITLs reflects the separate, quite well-established schools of thought which have nevertheless over the years maintained contact and arranged to meet from time to time. As already noted above, ITLs are often regarded as being either conceptually or computationally too complex for practical deployment. Somewhat paradoxically, researchers of pure formalisms (which are normally propositional) consider local ones too limited in scope and too close to point-based notations for their research purposes. This is because propositional versions of local formalisms are not considered suitable frameworks for properly expressing temporal phenomena such as accomplishments, durative actions and temporal aggregations (although such concepts can certainly also be relevant in formulas about intervals in local notations). Della Monica et al. [8] show that the addition of past-time operators to a pure logic can have a bigger effect on expressiveness than their addition to a conventional point-based logic has.

Another noteworthy division concerns DC, which, as already mentioned above, is intended for real-time and hybrid systems and is based on conventional ITL. In practice, work on DC and ITL has formed two distinct schools, like the ones for pure and local formalisms. Publications about DC often briefly mention the roots in ITL without further discussing links. This is so even for discrete-time DC [37, Sects. 6.1-6.2], which seems closely related to conventional ITL (e.g., both employ discrete time and support timing constraints). Furthermore, reductions of DC specifications to "implementables" (e.g., as described in [34]) involving automata seem to have potential connections with ITL. Perhaps in the future a holistic and systematic investigation and classification could be carried out to better understand and exploit natural connections and reductions with relevance 
to expressiveness, computational complexity, axiomatisation, executable subsets and other issues.

It is worthwhile to mention here that most research on ITLs is presently done in Europe. For instance, the special issue's authors are located in Denmark, Germany, Italy and the UK, but active researchers are also found in Bulgaria and Poland. However, China also has groups investigating ITLs. Furthermore, ITLs were high on the research and training agenda in formal methods for over a decade starting from 1994 at the United Nations University International Institute for Software Technology (UNU-IIST) in Macao. ${ }^{4}$ Activity in North America is presently on a significantly smaller scale than in Europe or China.

\section{Papers in the special issue}

Below are summaries of the articles appearing in this special issue:

- Two companion papers The light side of Interval Temporal Logic: the BernaysSchönfinkel fragment of CDT by Davide Bresolin, Dario Della Monica, Angelo Montanari and Guido Sciavicco,

and The dark side of Interval Temporal Logic: marking the undecidability border by Davide Bresolin, Dario Della Monica, Valentin Goranko, Angelo Montanari and Guido Sciavicco:

These are part of an extensive series of publications (see [21] for a bibliography) by essentially the same group of researchers, and document their recent successes in a sustained effort to get as close as possible to both sides of the border between decidability and undecidability of the pure propositional ITLs with modal operators which can express Allen's interval relations. As in their previous work, where the authors concentrate on neighbourhood modalities, both sublanguages and important classes of linear orders serving as the models of time are covered. The "light-side" paper presents, amongst other things, an insightful application of the Bernays-Schönfinkel class, a long-known decidable fragment of first-order logic, to obtain a new decidable subset of the standard interval temporal logic CDT. In contrast, the "dark-side" paper describes a tour de force of technical effort in the quest for negative results, with the key technique being an encoding of unsolvable tiling problems.

- Interval-based temporal functional dependencies: Specification and verification by Carlo Combi and Pietro Sala:

This contribution extends an existing point-based temporal framework for integrity constraints in temporal databases to work with intervals and Allen's relations. New algorithms based on B-trees provide the means to check that an update to such a relation preserves the integrity constraints. Unlike the formalisms in other articles in the special issue, the framework is not modal, thus reflecting current practice in applications of intervals to databases.

- RGITL: A Temporal logic framework for compositional reasoning about interleaved programs by Gerhard Schellhorn, Bogdan Tofan, Gidon Ernst, Jörg Pfähler and Wolfgang Reif:

This paper, which involves the down-to-earth world of local formalisms, offers a self-contained presentation of another long-term effort: achieving rely-guarantee

\footnotetext{
${ }^{4}$ One of this special issue's guest editors, Dimitar Guelev, takes this opportunity to gratefully acknowledge that he developed his interest in DC during a fellowship at UNU-IIST.
} 
deductive verification of interleaved multithreaded programs using the interactive theorem prover KIV developed by the authors' research group. A key insight is the semantics for interleaving underlying an executable binary operator added to ITL. The operator's conceptual complexity exceeds that of basic ITL modalities by an order of magnitude. Nevertheless, its use in symbolic execution of programs represented as ITL formulas fits well with other constructs from executable ITL subsets such as Tempura. The authors note the framework's advantages over conventional point-based temporal logics in deductive proofs for concurrent algorithms they have considered.

- Compositional reasoning using intervals and time reversal by Ben Moszkowski:

Natural links between point- and interval-based temporal logics play a major role in this paper. In particular, various mathematically quite simple but previously unknown classes of sequentially compositional temporal formulas, including many point-based ones, are shown to be closed under both logical conjunction and a conventional pointbased operator. Symmetry techniques for viewing formulas' behaviour in reverse help to then prove the membership of more formulas in the classes. An analysis of concrete and abstract concurrent share-variable algorithms for mutual exclusion helps illustrate how the compositional formulas can be used.

- A practical approach to model checking Duration Calculus using Presburger Arithmetic by Michael R. Hansen, Anh-Dung Phan and Aske W. Brekling:

Two relatively independent research directions discussed in this article are combined to lower the forbidding complexity of validity and model checking of DC formulas with many alternations between chop and negation. In one direction a semantics for discrete-time DC based on Parikh images provides a useful abstraction of the standard DC semantics. The other direction concerns limiting the size and complexity of (reasoning with) Presburger arithmetic formulas obtained from a translation from DC to them with the abstract semantics. Both aspects of the work are very practically motivated. The reader is offered a variety of applicable techniques and judgements from real-life model checking and automated verification.

\begin{abstract}
Acknowledgments The guest editors would like to thank the AMAI Editor-in-Chief Professor Martin Golumbic for agreeing to have the special issue, and for his encouragement and support from beginning until end. In addition, Ms Belle Mojado of Springer's Journals Editorial Office showed greatly appreciated professionalism in her kind help with the management of the submissions during the various stages of processing and offered valuable advice throughout. It goes without saying that the special issue and its associated TIME 2011 conference special track would not have happened without the considerable efforts of the papers' authors. Also indispensable were the TIME steering committee masterfully headed by Professor Angelo Montanari and of course the TIME 2011 organisers and committees, as well as others who reviewed conference submissions. A special thanks is reserved for the reviewers of the special issue's submissions for making careful assessments and providing constructive advice to the authors and guest editors. These reviewers' names are listed below, with the exception of the ones for Ben Moszkowski's article:
\end{abstract}

Benedikt Bollig, Catalin Dima, Emmanuel Filiot, Marcelo Finger, Carlo A. Furia, Peter Höfner, Arjen Hommersom, Cliff Jones, Emanuel Kieroński, Kamal Lodaya, Jakub Michaliszyn, Gerhard Schellhorn, Dmitry Shkatov, Richard T. Snodgrass, Qiwen Xu and Naijun Zhan.

\title{
References
}

1. Allen, J.F.: Maintaining knowledge about temporal intervals. Commun. ACM 26(11), 832-843 (1983)

2. Banieqbal, B., Barringer, H.: Temporal logic with fixed points. In: Banieqbal, B., Barringer, H., Pnueli, A. (eds.) Temporal Logic in Specification, Proceedings (Altrincham, UK, April, 1987), LNCS, vol. 398, pp. 62-74. Springer, Berlin (1989) 
3. Barringer, H., Fisher, M., Gabbay, D., Gough, G.: Owens, R.: MetateM: A framework for programming in temporal logic. In: J. Bakker, W.P. Roever, G. Rozenberg (eds.) Stepwise Refinement of Distributed Systems: Models, Formalisms, Correctness (REX Workshop, 1989), no. 430 in LNCS, pp. 94-129. Springer (1990)

4. Bäumler, S., Schellhorn, G., Tofan, B., Reif, W.: Proving linearizability with temporal logic. Form. Asp. Comput 23(1), 91-112 (2011)

5. Bresolin, D., Della Monica, D., Montanari, A., Sciavicco, G.: A tableau system for right propositional neighborhood logic over finite linear orders: an implementation. In: D. Galmiche, D. Larchey-Wendling (eds.) Proceedings of the 22th International Conference on Automated Reasoning with Analytic Tableaux and Related Methods (TABLEAUX 2013), LNCS, vol. 8123, pp. 74-80. Springer (2013)

6. Dam, M.: Temporal logic, automata, and classical theories: An introduction. Notes for the 6th European Summer School in Logic, Language, and Information (ESSLLI 1994, Copenhagen) (1994). http://web. it.kth.se/mfd/Papers/tlact.pdf. Accessed 19 December 2013

7. Della Monica, D., Goranko, V., Montanari, A., Sciavicco, G.: Interval temporal logics: a journey. Bull. EATCS 105, 73-99 (2011)

8. Della Monica, D., Montanari, A., Sala, P.: The importance of the past in interval temporal logics: the case of propositional neighborhood logic. In: A. Artikis, R. Craven, N.K. Çiçekli, B. Sadighi, K. Stathis (eds.) Logic Programs, Norms and Action, LNCS, vol. 7360, pp. 79-102. Springer (2012)

9. Dutertre, B.: Complete proof systems for first order interval temporal logic. In: Proceedings of the 10th Annual IEEE Symposium on Logic in Computer Science (LICS '95), pp. 36-43. IEEE Computer Society Press, Los Alamitos (1995)

10. Fisher, M.: An Introduction to Practical Formal Methods Using Temporal Logic. Wiley (2011)

11. Golumbic, M.C., Shamir, R.: Complexity and algorithms for reasoning about time: A graph-theoretic approach. J. ACM 40(5), 1108-1133 (1993)

12. Goranko, V., Montanari, A.: Foreword to special issue on interval temporal logics and duration calculi. J. Appl. Non-Classical Log. 14(1-2), 7-8 (2004)

13. Goranko, V., Montanari, A., Sciavicco, G.: A road map of propositional interval temporal logics and duration calculi. J. Appl. Non-Classical Log. 14(1-2), 9-54 (2004)

14. Gordon, M.J.C.: From LCF to HOL: A short history. In: G. Plotkin, C. Stirling, M. Tofte (eds.) Proof, Language, and Interaction: Essays in Honour of Robin Milner, pp. 169-185. MIT Press, Cambridge (2000)

15. Halpern, J.Y., Shoham, Y.: A propositional modal logic of time intervals. J. ACM 38(4), 935-962 (1991)

16. Hansen, M.R., Zhou, C.: Duration calculus: logical foundations. Form. Asp. Comput. 9(3), 283-330 (1997)

17. Hoare, T.: Verification of fine-grain concurrent programs. Electr. Notes Theor. Comput. Sci. 209, 165171 (2008)

18. IEEE: Standard for the Functional Verification Language e, Standard 1647-2011. ANSI/IEEE, New York (2011)

19. ISO: ISO/IEC 9075-2:2011 Information technology — Database languages — SQL — Part 2: Foundation (SQL/Foundation). ISO, Geneva (2011)

20. Interval Temporal Logic website at De Montfort University, UK. http://www.tech.dmu.ac.uk/STRL/ITL/. Accessed 24 April 2013

21. Interval Temporal Logics website at the University of Udine, Italy. http://itl.dimi.uniud.it/content/ logic-hs and http://itl.dimi.uniud.it/biblio. Accessed 27 December 2013

22. Jones, C.B.: Tentative steps toward a development method for interfering programs. ACM Trans. Progr. Lang. Syst. 5(4), 596-619 (1983)

23. Jones, C.B.: Balancing expressiveness in formal approaches to concurrency. Tech. Rep. 1394, University of Newcastle. Department of Computing Science (2013). http://www.cs.ncl.ac.uk/publications/trs/ papers/1394.pdf. Accessed 10 December 2013

24. Kröger, F., Merz, S.: Temporal Logic and State Systems. Springer (2008)

25. Kulkarni, K., Michels, J.E.: Temporal features in SQL:2011. ACM SIGMOD Rec. 41(3), 34-43 (2012)

26. Lamport, L.: Specifying Systems: The TLA+ Language and Tools for Hardware and Software Engineers. Addison-Wesley Professional (2002)

27. Lichtenstein, O., Pnueli, A., Zuck, L.: The glory of the past. In: R. Parikh (ed.) Logics of Programs, LNCS, vol. 193, pp. 196-218. Springer (1985)

28. Lomuscio, A., Michaliszyn, J.: An epistemic Halpern-Shoham logic. In: F. Rossi (ed.) Proceedings of the 23rd International Joint Conference on Artificial Intelligence (IJCAI 2013) (2013)

29. Manna, Z., Pnueli, A.: The Temporal Logic of Reactive and Concurrent Systems: Specifications. Springer, New York (1992) 
30. Moszkowski, B.: Reasoning about digital circuits. Ph.D. thesis, Tech. Rep. STAN-CS-83-970. Department of Computer Science, Stanford University (1983)

31. Moszkowski, B.: Executing Temporal Logic Programs. Cambridge University Press. Cambridge (1986)

32. Moszkowski, B.: A hierarchical completeness proof for propositional interval temporal logic with finite time. J. Appl. Non Class Log. 14(1-2), 55-104 (2004)

33. Müller-Olm, M.: A modal fixpoint logic with chop. In: C. Meinel, S. Tison (eds.) Proceedings of the 16th Annual Symposium on Theoretical Aspects of Computer Science (STACS 99), LNCS, vol. 1563, pp. 510-520. Springer (1999)

34. Olderog, E.R., Dierks, H.: Real-Time Systems: Formal Specification and Automatic Verification. Cambridge University Press. Cambridge (2008)

35. Roy, S., Chaochen, Z.: Notes on Neighbourhood Logic. Tech. Rep. 97. UNU-IIST, Macao (1997)

36. Venema, Y.: A modal logic for chopping intervals. J. Log. Comput. 1(4), 453-476 (1991)

37. Zhou, C.: Hansen, M.R.: Duration calculus: A Formal Approach to Real-Time Systems. Springer (2004)

38. Zhou, C., Hoare, C.A.R., Ravn, A.P.: A calculus of durations. Inf. Process. Lett. 40(5), 269-276 (1991) 